\title{
Exceptional Optoelectronic Properties of Hydrogenated Bilayer Silicene
}

\author{
Bing Huang, ${ }^{1,2, *}$ Hui-Xiong Deng, ${ }^{3}$ Hoonkyung Lee, ${ }^{4}$ Mina Yoon, ${ }^{2}$ Bobby G. Sumpter, ${ }^{2}$ Feng Liu, ${ }^{5}$ \\ Sean C. Smith, ${ }^{2}$ and Su-Huai Wei ${ }^{1, \dagger}$ \\ ${ }^{1}$ National Renewable Energy Laboratory, Golden, Colorado 80401, USA \\ ${ }^{2}$ Center for Nanophase Materials Sciences, Oak Ridge National Laboratory, Oak Ridge, \\ Tennessee 37831-6493, USA \\ ${ }^{3}$ State Key Laboratory for Superlattices and Microstructures, Institute of Semiconductors, Chinese \\ Academy of Sciences, P.O. Box 912, Beijing 100083, China \\ ${ }^{4}$ Division of Quantum Phases and Devices, School of Physics, Konkuk University, Seoul 143-701, Korea \\ ${ }^{5}$ Department of Materials Science and Engineering, University of Utah, Salt Lake City, Utah 84112, USA \\ (Received 25 November 2013; revised manuscript received 7 April 2014; published 19 May 2014)
}

\begin{abstract}
Silicon is arguably the best electronic material, but it is not a good optoelectronic material. By employing first-principles calculations and the cluster-expansion approach, we discover that hydrogenated bilayer silicene (BS) shows promising potential as a new kind of optoelectronic material. Most significantly, hydrogenation converts the intrinsic BS, a strongly indirect semiconductor, into a direct-gap semiconductor with a widely tunable band gap. At low hydrogen concentrations, four ground states of single- and doublesided hydrogenated BS are characterized by dipole-allowed direct (or quasidirect) band gaps in the desirable range from 1 to $1.5 \mathrm{eV}$, suitable for solar applications. At high hydrogen concentrations, three well-ordered double-sided hydrogenated BS structures exhibit direct (or quasidirect) band gaps in the color range of red, green, and blue, affording white light-emitting diodes. Our findings open opportunities to search for new silicon-based light-absorption and light-emitting materials for earth-abundant, highefficiency, optoelectronic applications.
\end{abstract}

DOI: 10.1103/PhysRevX.4.021029

Silicon is one of the most important building blocks in today's high-technology fields. For example, the majority of solar cells fabricated to date have been based on singlecrystalline or polycrystalline $\mathrm{Si}$ in a diamond structure, with a market share of greater than $90 \%$ [1]. This is not only because $\mathrm{Si}$ is the second-most-abundant element on the Earth but also because the Si-based photovoltaic (PV) industry benefits from the mature Si-based microelectronics technology. However, although diamond $\mathrm{Si}$ has a fundamental (indirect) band gap of $1.12 \mathrm{eV}$ [2], its optical (direct) gap is larger than $3 \mathrm{eV}$ [3]; thus, $\mathrm{Si}$ is not a good absorber of sunlight [4]. As a result, the $\mathrm{Si}$ absorber layer must be sufficiently thick (usually $>100 \mu \mathrm{m}$ ) to absorb enough visible light, and it must be assisted by phonons, which significantly increases the cost of Si-based PV modules [5]. Because of this drawback, in the past several decades, significant effort has been devoted to the search of materials that have better absorption coefficients in the visible region. One approach is to identify

\footnotetext{
*huangb@ornl.gov

†suhuai.wei@nrel.gov
}

Published by the American Physical Society under the terms of the Creative Commons Attribution 3.0 License. Further distribution of this work must maintain attribution to the author(s) and the published article's title, journal citation, and DOI.
Subject Areas: Computational Physics,

Condensed Matter Physics, Graphene

different materials that can replace silicon for solar cells. For example, CdTe [6,7], $\mathrm{Cu}(\mathrm{In}, \mathrm{Ga}) \mathrm{Se}_{2}[8,9]$, and $\mathrm{Cu}_{2} \mathrm{ZnSn}(\mathrm{S}, \mathrm{Se})_{4}[10,11]$ based single-junction, thin-film solar cells have been widely studied in the past few decades because these absorbers have promising direct band gaps between 1 and $1.5 \mathrm{eV}$, which are expected to produce highefficiency solar cells according to the Shockley-Queisser criteria [12]. Another approach would be to engineer the structural and electronic properties of silicon to improve its optical absorption.

Several specific approaches have been proposed to manipulate the band structure and optical property of silicon. The first method is to search metastable silicon phases with better optical coefficients in the visible region. For example, cubic $\mathrm{Si}_{20}$ [13] and M-10 Si [14] are predicted to have quasidirect band gaps of 1.40 and $1.55 \mathrm{eV}$, respectively. However, the synthesis of these high-energy metastable silicon phases is still a challenge. The second method is to alloy Si with other common materials to obtain new functional materials. For example, alloying $\mathrm{Si}$ with AlP to form $\mathrm{Si}_{3} \mathrm{AlP}$ [15] can reduce the optical gap of $\mathrm{Si}$ to around $2.0 \mathrm{eV}$ [16], which is much smaller than that of diamond Si but still higher than the optimal value for solarcell application; alloying $\mathrm{Si}$ with $\mathrm{Ge}$ on a proper substrate may form the magic superlattice structures such as $\alpha_{12}$ Si-Ge with a dipole-allowed direct band gap of $0.61 \mathrm{eV}$ $[17,18]$, but experimental synthesis of this complex 
structure is difficult because of the interface strain. The third method is to design proper nanostructured silicon, but the dipole transitions at the band edges of silicon quantum dots are quite weak and depend strongly on the structures and surface passivations [19]. These limitations encourage us to investigate new approaches to manipulate the electrical and optical properties of silicon materials for optoelectronic applications.

Recently, a new kind of two-dimensional (2D) hexagonal silicon structure, silicene, has attracted significant attention because it has a graphenelike structure and similar electronic bands, e.g., a Dirac cone around the Fermi energy [20-22]. The experimental progress demonstrates that single- and few-layer silicene can be synthesized by epitaxial growth on several substrates, e.g., Ag [23-32], Ir [33], and $\mathrm{ZrB}_{2}$ [34]. The freestanding bilayer silicene (BS), which is more stable than single-layer silicene, has been predicted to be an ideal material for topological insulators or superconductors [35,36] and Li-ion storage [37]. Although bilayer silicene has been synthesized in several experiments $[24,26,28-32]$, the ground-state structure of bilayer silicene, even in its freestanding form, is still under debate [35,37-39].

Low-dimensional materials themselves are usually not sufficient for various applications, and chemical functionalization can play an important role to further modify their electronic properties for various specific applications. Among numerous chemical methods, hydrogenation has been demonstrated to be one of the most powerful approaches to tune the electronic properties of 2D materials. For example, it has been demonstrated that hydrogenation can open sizable band gaps in graphene [40-42], graphyne [43], and single-layer silicene [44-46]. However, none of these hydrogenated structures reported to date is suitable for solar applications; in particular, there may be a strong phase separation in the ground states of these materials. As a new silicon phase, it is quite interesting to study if the intrinsic and hydrogenated BS (HBS) could realize controllable optoelectronic properties for solar cells and other optical applications.

In this article, by employing first-principles calculations and the cluster-expansion approach, we have systemically studied the structural and electronic properties of intrinsic and hydrogenated BS. Our calculations demonstrate that BS is a strongly indirect semiconductor that is not suitable for optoelectronics. However, hydrogenation of BS can induce some well-ordered intermediate phases with unexpected electronic properties. At low hydrogen concentrations, four single- and double-sided HBS ground states have dipole-allowed direct (or quasidirect) band gaps with gaps between 1 and $1.5 \mathrm{eV}$, which are suitable for solar applications. Surprisingly, at high hydrogen concentrations, we discover three well-ordered double-sided HBS structures that have direct (or quasidirect) band gaps within the color range of red, green, and blue (RGB). The combination of RGB emitters can achieve a white lightemitting diode (LED), which has great potential for solidstate lighting. Thus, hydrogenation of BS can achieve new silicon-based solar absorbers, but it can also extend silicon to other optoelectronic applications such as white LEDs.

All the density-functional-theory (DFT) calculations are performed by using the VASP code [47]. Projectoraugmented-wave (PAW) potentials are used to describe the core electrons, and the generalized gradient approximation (GGA) with the Perdew-Burke-Ernzerhof (PBE) functional is selected in our calculations. The effect of van de Waals ( $\mathrm{vdW}$ ) interactions is taken into account by using the empirical correction scheme of Grimme (DFT $+\mathrm{D} / \mathrm{PBE}$ ) [48], which has proved to be successful in describing various layered structures [49,50]. The kinetic-energy cutoff for the plane-wave basis is set to $400 \mathrm{eV}$. A $15-\AA$ vacuum layer is adopted to simulate isolated bilayer silicene. The $\Gamma$-centered $k$-point mesh with sufficient $k$-point density is used for the Brillouin-zone integration for all the structures. All the structures are fully relaxed until the force on each atom is less than $0.01 \mathrm{eV} / \AA$. It is well known that normal DFT calculations usually underestimate the band gaps of semiconductors. Because hybrid functional calculations could give improved results for both conventional semiconductors and low-dimensional materials, we adopt the Heyd, Scuseria, and Ernzerhof (HSE06) hybrid functional [51] to calculate all the electronic structures of our studied systems. It has been shown that the HSE06 functional can describe the band gaps of new silicon phases very well, close to GW results $[13,14]$.

To search the ground-state structures at different hydrogen concentrations, we treat $\mathrm{HBS}\left(\mathrm{SiH}_{x}\right)$ as alloy systems, with each site either occupied by hydrogen or not, and we use the cluster-expansion (CE) method established in the alloy theory to describe the system. In the CE approach, the alloy Hamiltonian is mapped onto a generalized Ising Hamiltonian [52,53]:

$$
\begin{aligned}
E(\sigma)= & J_{0}+\sum_{i} J_{i} \hat{S}_{i}(\sigma)+\sum_{j<i} J_{i j} \hat{S}_{i}(\sigma) \hat{S}_{j}(\sigma) \\
& +\sum_{k<j<i} J_{i j k} \hat{S}_{i}(\sigma) \hat{S}_{j}(\sigma) \hat{S}_{k}(\sigma)+\cdots,
\end{aligned}
$$

where the indices $i, j$, and $k$ range over all occupation sites. $S_{m}(\sigma)$ is +1 when it is occupied by $\mathrm{H}$ and -1 if it is not. Every cluster figure $\alpha$ is associated with an interaction parameter $J_{\alpha}$, which gives the contribution of the specific cluster figure to the properties. Generally, CE is able to represent any $\mathrm{SiH}_{x}$ energy $E(\sigma)$ by an appropriate selection of $J_{\alpha}$, and then it can predict the absolute lowest-energy structures within certain atom supercells. The parameters of $J_{\alpha}$ can be determined by fitting them to the first-principles energies of selected configurations [54]. Initially, we put $\mathrm{H}$ atoms randomly on the top site of bilayer silicene to generate various alloy structures. The cross-validation 
(CV) score [54], obtained by a least-squares fit to firstprinciples energies, is used to measure the CE quality, and we have made sure that all the CE fittings in our study can reach a reasonably low $\mathrm{CV}$ score.

For the stacking of two single-layer silicene, there are generally two types of BS with high symmetry, i.e., AA and $\mathrm{AB}$ stacking. Because of the existence of buckling in the single-layer structure $[22,46]$, the single-layer silicene can be divided into top and bottom Si layers. This nonplanar structure allows each $\mathrm{AA}$ and $\mathrm{AB}$ stacking to have two nonequivalent variants (top-top and top-down); i.e., there are four possible stacking structures with different symmetries: AA-1 ( $\mathrm{D}_{3 d}$ symmetry), AA-2 ( $\mathrm{D}_{3 h}$ symmetry), $\mathrm{AB}-1 \quad\left(\mathrm{D}_{3 d}\right.$ symmetry), and $\mathrm{AB}-2\left(\mathrm{C}_{3 v}\right.$ symmetry), as shown in Fig. 1(a). The formation energy $\mathrm{E}_{f}$ of bilayer silicene with respect to single-layer silicene is defined as $E_{f}=\left(E_{\mathrm{BS}}-2 E_{S}\right) / 4$, where $\mathrm{E}_{\mathrm{BS}}$ and $\mathrm{E}_{S}$ are the total energies (per unit cell) of bilayer and single-layer silicene, respectively. Obviously, all these bilayer structures have lower energies than that of single-layer silicene. In these four structures, $\mathrm{AB}-1$ has the lowest energy, which is consistent with the conclusion from Liu et al. [35]. However, our further investigation finds that the AA-1 and AA-2 structures are both metastable, and the most stable AA-stacking structure is AA-3 with $\mathrm{D}_{6 h}$ symmetry [Fig. 1(a)]. For the structure of AA-3, the in-plane Si-Si bond length is stretched from an initial $2.25 \AA$ to $2.39 \AA$, and the silicene layers become flat. At the same time, the interlayer Si-Si bond lengths are decreased to $2.42 \AA$, which means that all the Si atoms form interlayer bonds to fully realize $\mathrm{sp}^{3}$ hybridization. As a result, the $\mathrm{E}_{f}$ of the AA-3 structure is $24 \mathrm{meV} / \mathrm{Si}$ lower than that of AB-1. This conclusion does not change even when excluding the vdW corrections. Our finding is consistent with that from Tritsaris et al. [37] based on PBE calculations without $\mathrm{vdW}$ corrections. To further enhance our conclusion, we have performed global-minimum optimization using the particle-swarm-optimization (PSO) method [55] to confirm the proposed bilayer AA-3 structure as the global ground state. In this structure search, the hexagonal lattice of $\mathrm{Si}$ is not assumed. Instead, we generate random structures (both atomic coordinates and lattice parameters) to initialize the simulations. The population size is set to 20 , and the number of generations is set to 20 . The number of atoms is fixed to $4 \mathrm{Si} /$ cell, and only bilayer structures are considered. After several generations, we have successfully confirmed that the AA-3 structure is indeed the most stable bilayer silicon phase.

It is important to check the stability of the bilayer AA-3 structure. First, the phonon spectrum of AA-3 is calculated by finite-different methods to understand its dynamic stability. As demonstrated in Fig. 1(b), there are no imaginary phonon modes in the whole Brillouin zone, which indicates that the AA-3 structure is dynamically

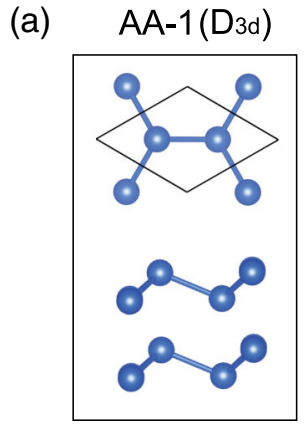

$E_{f}=-186 \mathrm{meV} / \mathrm{Si}$

(b)

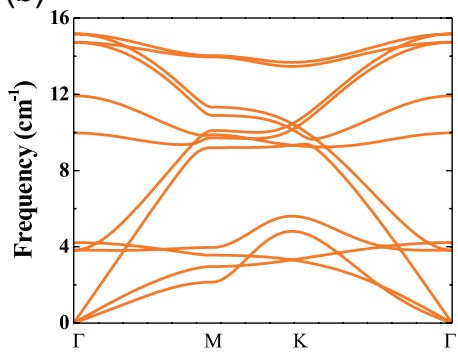

AA-2 (D3h)

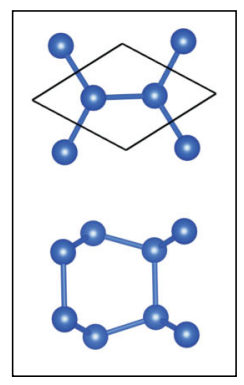

$E_{f}=-162 \mathrm{meV} / \mathrm{Si}$

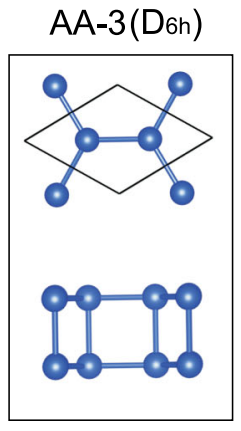

$E_{f}=-218 \mathrm{meV} / \mathrm{Si}$

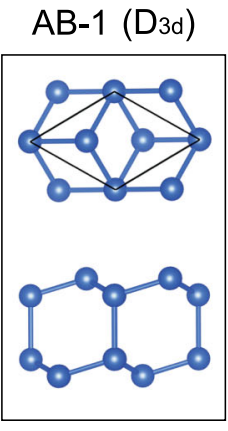

$E_{f}=-194 \mathrm{meV} / \mathrm{Si}$

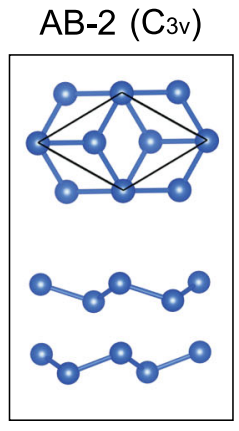

$E_{f}=-107 \mathrm{meV} / \mathrm{Si}$ (c)

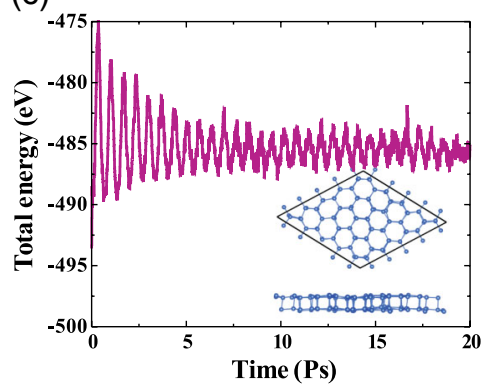

(d)

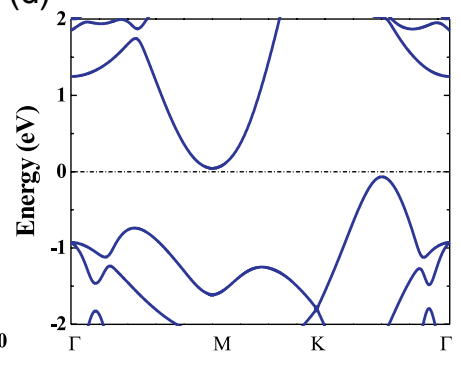

FIG. 1. (a) The structure and symmetry of five BS structures with AA or AB stacking. The formation energy $\mathrm{E}_{f}$ of each structure with respect to single-layer silicene is also plotted. (b) The phonon spectrum of the AA-3 structure. (c) The fluctuation of total energy of (100-atoms/cell) the AA-3 structure as a function of the molecular-dynamic simulation step at $600 \mathrm{~K}$. (d) The calculated band structure of the AA-3 structure. 
stable. Second, first-principles molecular-dynamic simulations, using a Nose-Hoover thermostat with a time step of $2 \mathrm{fs}$, are performed to check the thermal stability of AA-3 at a finite temperature. A $5 \times 5$ 100-atom AA-3 supercell is selected, as shown in the inset of Fig. 1(c). The fluctuation of the total energy as a function of simulation time at $600 \mathrm{~K}$ is plotted in Fig. 1(c). After 20 ps, no structural destruction of AA-3 is found, except for some thermal fluctuations, which demonstrates that freestanding AA-3 can stably exist (at least) at $600 \mathrm{~K}$. This temperature is high enough for optoelectronic applications. Unfortunately, the calculated band structure [Fig. 1(d)] shows that the bilayer AA-3 is a strongly indirect semiconductor. It has a minimum direct band gap of $1.6 \mathrm{eV}$ (at the $\mathrm{M}$ point) and a fundamental band gap of $0.10 \mathrm{eV}$ [a conduction-band minimum (CBM) at the $\mathrm{M}$ point and valence-band maximum (VBM) at the $\mathrm{K}-\Gamma$ line]. We also note that the AA-3 structure shows metallic (negative band gap) behavior under conventional PBE calculations, indicating that hybrid functional calculations are quite necessary to correctly describe the electronic structures of BS-related materials. Although there are some experimentally measured band structures of BS on the $\mathrm{Ag}$ (111) surface, it is difficult to directly compare our theoretical results for freestanding BS to these experimental ones because the strong interactions between the $\mathrm{Si}$ and $\mathrm{Ag}$ (111) surface can largely modify the electronic structure of freestanding BS [27]. Like bulk silicon, this kind of strongly indirect band structure is not suitable for solar and other optoelectronic applications. Thus, it is highly desirable to investigate the possibility of modifying this new silicon structure by hydrogenation. In the present article, we focus our study on freestanding BS. For the cases of stable configurations of BS on substrates, e.g., an $\operatorname{Ag}(111)$ substrate, some discussions are provided in Ref. [56].

In practice, hydrogen atoms can adsorb on either single or double sides of BS during hydrogenation, depending on the situations of hydrogenation [40-42]. Thus, both singlesided and double-sided HBS are systemically studied. Because of the small size of $\mathrm{H}$ atoms, our calculations show that $\mathrm{H}$ atoms prefer to adsorb on the top of $\mathrm{Si}$ atoms, similar to hydrogenated graphene [40-42,57]. For singlesided $\mathrm{SiH}_{x}$, the highest $\mathrm{H}$ concentration is $x=0.5$. In the structure of $\mathrm{SiH}_{0.5}$ [inset of Fig. 2(a)], hydrogen-atom adsorption on the top basal plane of BS breaks the interlayer $\mathrm{Si}-\mathrm{Si}$ bonds, which indicates that the strength of $\mathrm{Si}-\mathrm{H}$ bonds is stronger than that of interlayer $\mathrm{Si}-\mathrm{Si}$ bonds. After the interlayer bonds are broken, the in-plane Si-Si bond lengths are decreased to $2.32 \AA$, while the interlayer $\mathrm{Si}-\mathrm{Si}$ bond lengths are increased to $3.34 \AA$, belonging to vdW interactions. For a single-sided $\mathrm{SiH}_{x}$ with arbitrary configuration $\sigma(x)$, its formation energy $\left(\mathrm{E}_{f}\right)$ is defined as

$$
E_{f}(\sigma)=E\left(\mathrm{SiH}_{x}\right)-(1-2 x) E\left(\mathrm{SiH}_{0}\right)-2 x E\left(\mathrm{SiH}_{0.5}\right),
$$

where $E\left(\mathrm{SiH}_{0}\right)$ and $E\left(\mathrm{SiH}_{0.5}\right)$ are the energies of silicene and $\mathrm{SiH}_{0.5}$, respectively. We have calculated the $\mathrm{E}_{f}$ of selected $80 \quad \mathrm{SiH}_{x}$ structures, containing at most 42 atoms/unit cell, using first-principles methods. The $\mathrm{CE}$ Hamiltonian of $\mathrm{SiH}_{x}$ is obtained by fitting to the 80 first-principles energies. The CV score of this CE fitting is $33 \mathrm{meV}$, indicating that the obtained $\mathrm{CE}$ Hamiltonian is accurate enough to predict the $\mathrm{E}_{f}$ of all the $\mathrm{SiH}_{x}$ structures. Then, the energies of all the symmetry-inequivalent $\mathrm{SiH}_{x}$ alloys (> 10000) within 42 atoms/cell are calculated by the CE Hamiltonian, and the results are shown in Fig. 2(a). Interestingly, six intermediate ground states are discovered, as plotted in Fig. 3 for $\mathrm{Si}_{16} \mathrm{H}_{2}\left(\mathrm{C}_{2 v}\right.$ symmetry $), \mathrm{Si}_{28} \mathrm{H}_{6}\left(\mathrm{C}_{1}\right.$ symmetry), $\mathrm{Si}_{24} \mathrm{H}_{6}\left(\mathrm{C}_{2}\right.$ symmetry), $\mathrm{Si}_{28} \mathrm{H}_{8}\left(\mathrm{C}_{2}\right.$ symmetry), $\mathrm{Si}_{12} \mathrm{H}_{4}\left(\mathrm{C}_{2 v}\right.$ symmetry), and $\mathrm{Si}_{16} \mathrm{H}_{6}\left(\mathrm{C}_{2 v}\right.$ symmetry). One common characteristic of these ground states is that the adsorbed $\mathrm{H}$ atoms on the top of some specific Si atoms break the underneath $\mathrm{Si}-\mathrm{Si}$ bonds and induce some local structural distortions. The average $\mathrm{Si}-\mathrm{Si}$ bond length
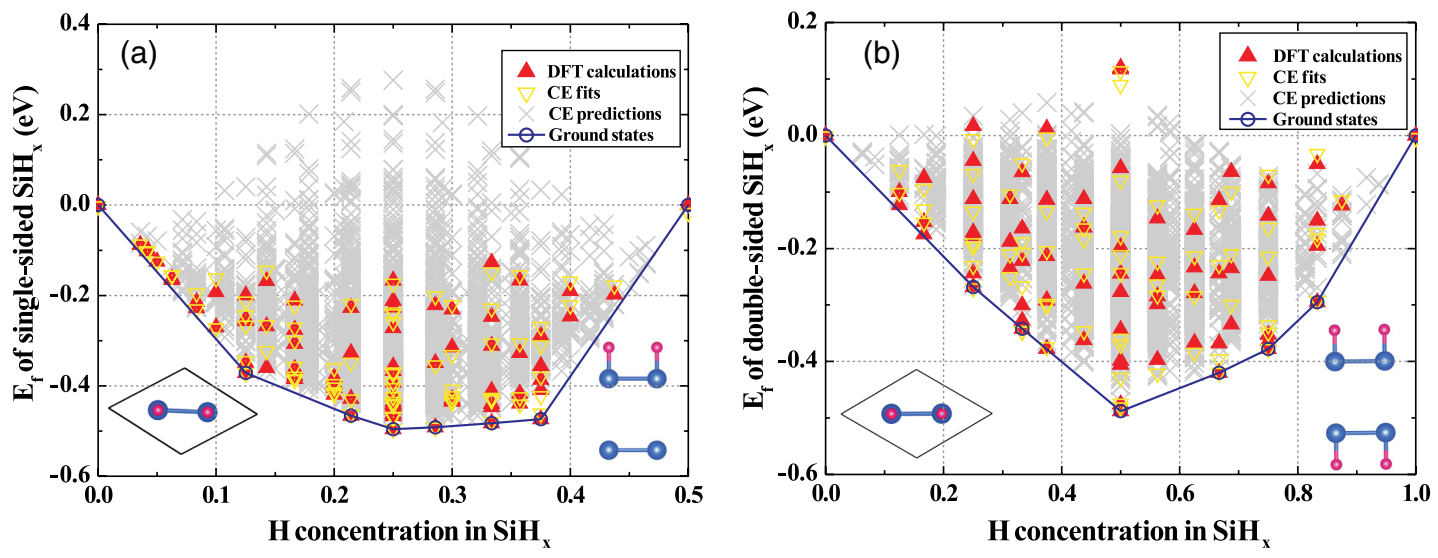

FIG. 2. (a) The calculated formation energies $\mathrm{E}_{f}$ of single-sided $\mathrm{SiH}_{x}$ along with the corresponding $\mathrm{CE}$ fits as a function of $x$. The $\mathrm{E}_{f}$ of all the symmetry-inequivalent structures calculated from $\mathrm{CE}$ are also plotted here. Inset: The top and side views of the structures of $\mathrm{SiH}_{0.5}$. Panel (b) is the same as (a) but for double-sided $\mathrm{SiH}_{x}$. The top and side views of the $\mathrm{SiH}_{1}$ structure are also shown in Fig. 2(b). 

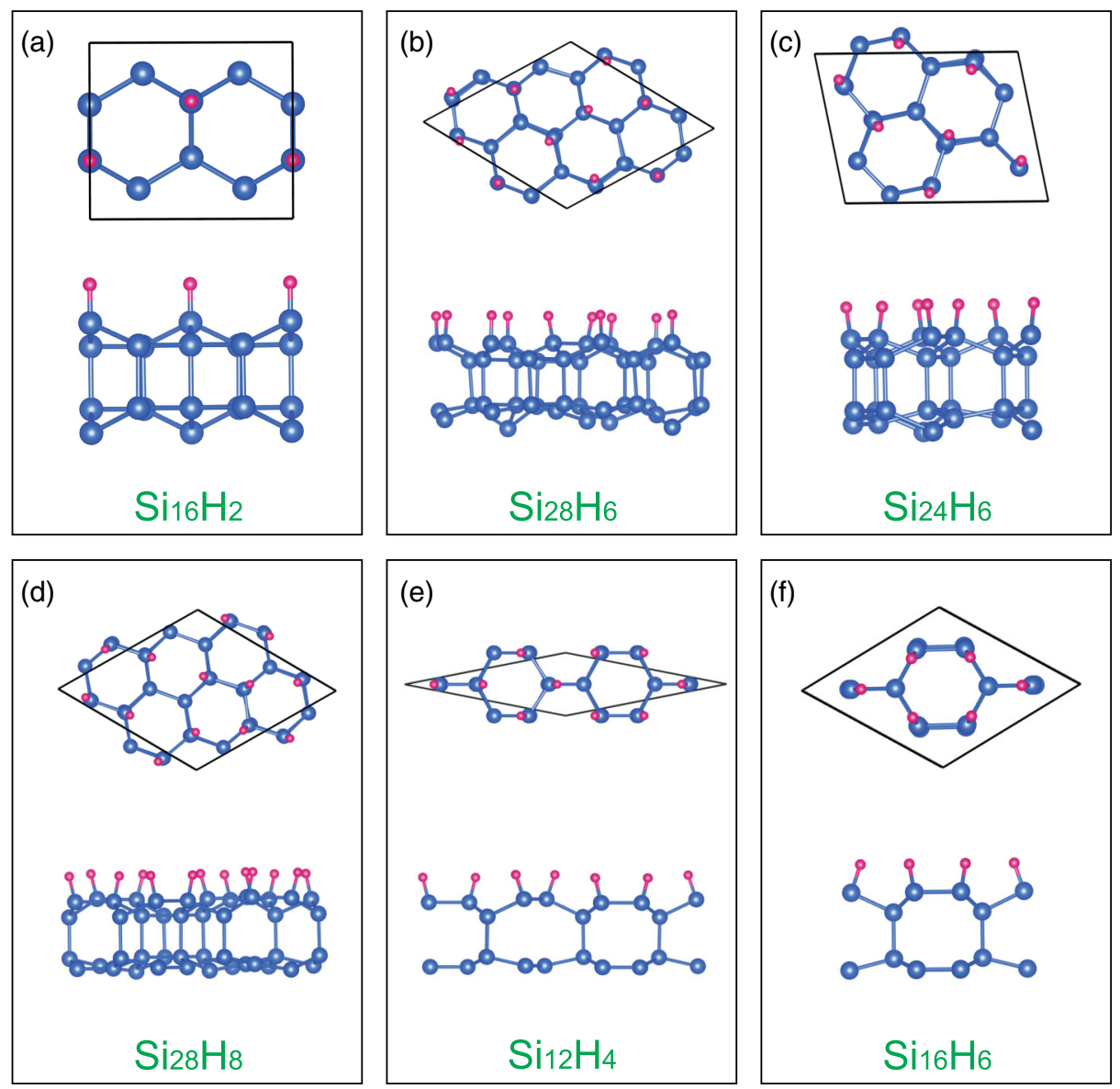

FIG. 3. The top and side views of six CE-discovered intermediate ground-state structures for single-sided (a) $\mathrm{Si}_{16} \mathrm{H}_{2}$, (b) $\mathrm{Si}_{28} \mathrm{H}_{6}$, (c) $\mathrm{Si}_{24} \mathrm{H}_{6}$, (d) $\mathrm{Si}_{28} \mathrm{H}_{8}$, (e) $\mathrm{Si}_{12} \mathrm{H}_{4}$, and (f) $\mathrm{Si}_{16} \mathrm{H}_{6}$.

$(2.34 \AA)$ and bond angle $\left(106^{\circ}\right)$ at the distortion sites are close to those in bulk $\mathrm{Si}$, which indicates that distortion can maximize the ideal $\mathrm{sp}^{3}$ hybridization to lower the total energies. Generally, the hexagonal sublattice of silicene is kept during hydrogenation, which is significantly different from that of hydrogenated single-layer silicene [46] and consistent with the fact that BS is more stable than singlelayer silicene.

For double-sided $\mathrm{SiH}_{x}$, the highest $\mathrm{H}$ concentration is $x=1$, and its structure is shown in Fig. 2(b). The $\mathrm{E}_{f}$ of double-sided $\mathrm{SiH}_{x}$ with configuration $\sigma(x)$ is defined as

$$
E_{f}(\sigma)=E\left(\mathrm{SiH}_{x}\right)-(1-x) E\left(\mathrm{SiH}_{0}\right)-x E\left(\mathrm{SiH}_{1}\right)
$$

where $E\left(\mathrm{SiH}_{0}\right)$ and $E\left(\mathrm{SiH}_{1}\right)$ are the energies of silicene and $\mathrm{SiH}_{1}$, respectively. The $\mathrm{E}_{f}$ of a total of 72 alloy structures are calculated using first-principles methods and fitted by the CE Hamiltonian. The CV score for this CE fitting is $36 \mathrm{meV}$. Using this CE Hamiltonian, we have calculated all the $\mathrm{E}_{f}$ of symmetry-inequivalent double-sided $\mathrm{SiH}_{x}$ structures (> 10000) within 36 atoms/cell, as shown in Fig. 2(b). Surprisingly, six intermediate ground states are discovered at specific $\mathrm{H}$ concentrations, which are quite different from chemically functionalized double-sided graphene [57]. Usually, there are no intermediate ground states in chemically functionalized freestanding $2 \mathrm{D}$ materials with single-atom thickness because the existence of strong double-sided adsorbate-adsorbate interactions can give rise to the clustering of adsorbates $[46,53,57]$. For double-sided HBS, the formation of the $s p^{3}$ bond on one side and the subsequent increase in the layer-layer distance significantly reduce the strength of the attraction of $\mathrm{H}$ on opposite sides. This explains why some intermediate ground states can form in double-sided HBS. As shown in Fig. 4, those structures are $\mathrm{Si}_{16} \mathrm{H}_{4}\left(\mathrm{D}_{6 h}\right.$ symmetry), $\mathrm{Si}_{12} \mathrm{H}_{4}\left(\mathrm{D}_{2 h}\right.$ symmetry), $\mathrm{Si}_{8} \mathrm{H}_{4}\left(\mathrm{D}_{2 h}\right.$ symmetry), $\mathrm{Si}_{12} \mathrm{H}_{8}\left(\mathrm{D}_{2 h}\right.$ symmetry), $\mathrm{Si}_{16} \mathrm{H}_{12}\left(\mathrm{D}_{2 h}\right.$ symmetry), and $\mathrm{Si}_{12} \mathrm{H}_{10}\left(\mathrm{D}_{3 h}\right.$ symmetry). In these six ground states, we find that $\mathrm{H}$ atoms prefer to symmetrically adsorb on the top and bottom sides of HBS, giving rise to relatively higher structural symmetry compared to single-sided HBS. Similar to single-sided $\mathrm{SiH}_{x}, \mathrm{H}$ adsorption breaks the underneath $\mathrm{Si}-\mathrm{Si}$ bonds, and the local distortion of $\mathrm{Si}$ atoms can maximize the ideal $\mathrm{sp}^{3}$ hybridization. Also, the average Si-Si bond length and bond angle at the distortion sites are close to those in single-sided HBS. The calculated average binding energy between $\mathrm{H}$ atoms 

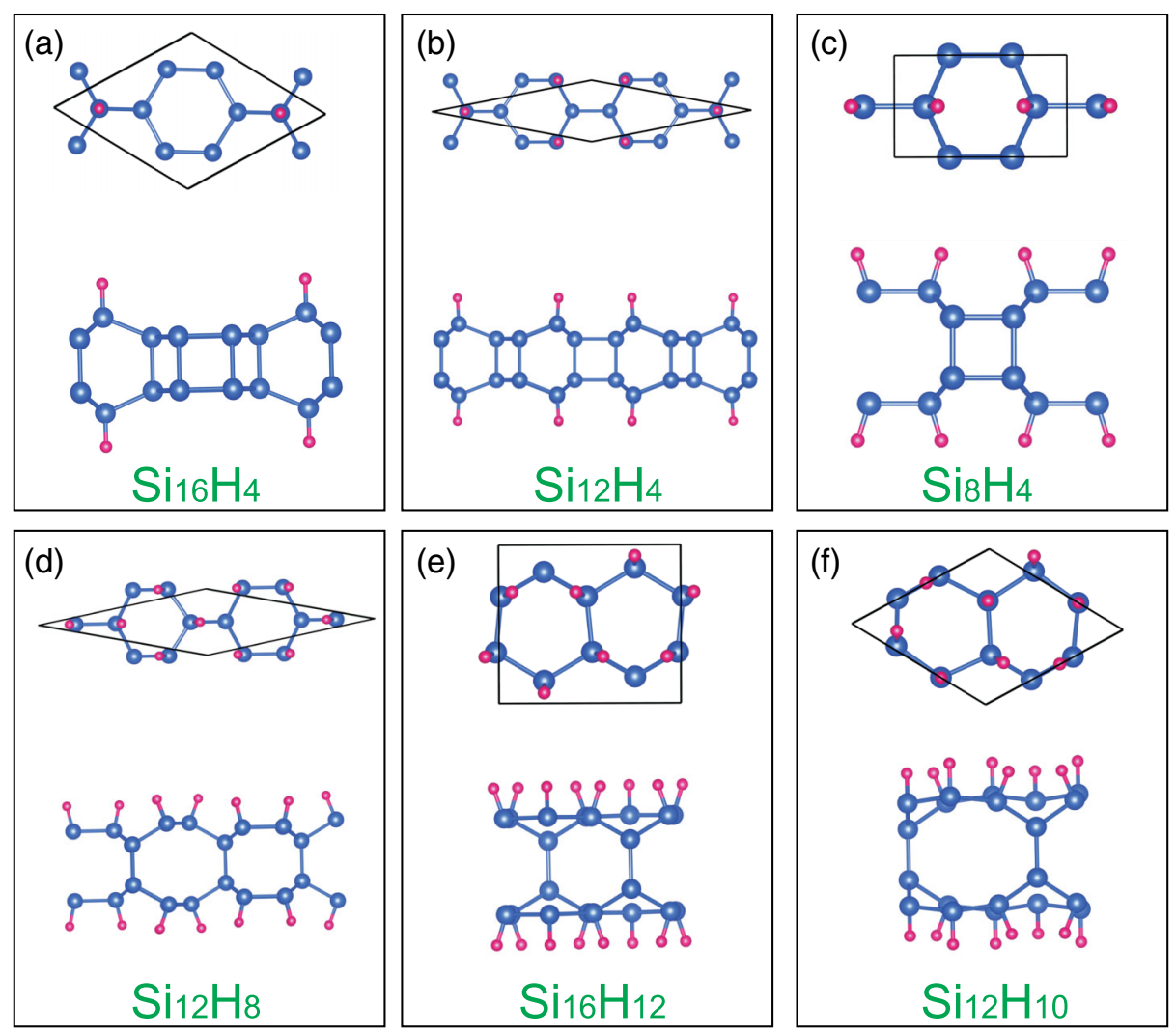

FIG. 4. The top and side views of six CE-discovered intermediate ground-state structures for double-sided (a) $\mathrm{Si}_{16} \mathrm{H}_{4},\left(\right.$ b) $\mathrm{Si}_{12} \mathrm{H}_{4}$, (c) $\mathrm{Si}_{8} \mathrm{H}_{4}$, (d) $\mathrm{Si}_{12} \mathrm{H}_{8}$, (e) $\mathrm{Si}_{16} \mathrm{H}_{12}$, and (f) $\mathrm{Si}_{12} \mathrm{H}_{10}$.

and BS in these ground states is around $820 \mathrm{meV} / \mathrm{H}$, close to the value between BS and the $\mathrm{Ag}(111)$ substrate, which indicates that hydrogenation plays a similar role as the $\mathrm{Ag}$ substrate to further stabilize the BS structures. Generally, the ground state of a single-or double-sided $\mathrm{SiH}_{x}$ structure with an arbitrary concentration could be phase separated into its neighboring ground states. For example, the ground state of any double-sided $\mathrm{SiH}_{0.4}$ structure can be the combination of $\mathrm{Si}_{12} \mathrm{H}_{4}$ [x=1/3, Fig. 4(b)] and $\mathrm{Si}_{8} \mathrm{H}_{4}$ $[x=1 / 2$, Fig. 4(c)] structures. This kind of phase separation is also experimentally observed in chemically functionalized graphene [58].

Because intrinsic BS is a strongly indirect-gap semiconductor, it is quite interesting to study the suitability of $\mathrm{SiH}_{x}$ for optoelectronics. In Fig. 5, we have plotted the calculated band gaps of these single-sided and double-sided $\mathrm{SiH}_{x}$ ground states as a functional of $\mathrm{H}$ concentration. For some special indirect band-gap semiconductors, if the gap differences between the indirect band gap and minimum direct band gap are less than $0.1 \mathrm{eV}$, we call them quasidirect gap semiconductors. Quasidirect band-gap systems may behave as direct band-gap systems in most optoelectronic applications [13,14,59]. As shown in Fig. 5, we find that there are large oscillations for the band gaps of single-sided $\mathrm{SiH}_{x}$ as a function of $\mathrm{H}$ concentration, which is significantly different from that of double-sided $\mathrm{SiH}_{x}$.
Single-sided $\mathrm{Si}_{28} \mathrm{H}_{6}$ and $\mathrm{Si}_{24} \mathrm{H}_{6}$ have direct (or quasidirect) band gaps of 1.01 and $1.30 \mathrm{eV}$, respectively, and doublesided $\mathrm{Si}_{16} \mathrm{H}_{4}$ and $\mathrm{Si}_{12} \mathrm{H}_{4}$ structures have direct (or quasidirect) band gaps of 1.52 and $1.46 \mathrm{eV}$, respectively. Clearly, these four $\mathrm{SiH}_{x}$ compounds have suitable band gaps for solar-absorbing applications.

Interestingly, we also found that double-sided $\mathrm{Si}_{8} \mathrm{H}_{4}$, $\mathrm{Si}_{12} \mathrm{H}_{8}, \mathrm{Si}_{16} \mathrm{H}_{12}$, and $\mathrm{Si}_{12} \mathrm{H}_{10}$ have the direct (quasidirect) band gaps of $1.85,1.99,2.43$, and $2.85 \mathrm{eV}$, respectively, which lie within red, orange, green, and blue colors, as shown in Fig. 5(b). Because $\mathrm{Si}_{8} \mathrm{H}_{4}, \mathrm{Si}_{16} \mathrm{H}_{12}$, and $\mathrm{Si}_{12} \mathrm{H}_{10}$ can realize the red, green, and blue colors, in a LED application, it may be possible to mix them in order to realize white color. Today, III-nitride materials, e.g., InGaN alloys, have became the frontrunner for achieving phosphors-free white LEDs via the combination of RGB emitters because the alloys with different compositions can, in principle, produce blue, green, and red LEDs $[60,61]$. However, it is known that it is exceedingly difficult to obtain red InGaN LEDs, and the efficiency of green LEDs using InGaN is also relatively low. This is partially because there is a large lattice mismatch $(>10 \%)$ between $\mathrm{GaN}$ and $\mathrm{InN}$, which can induce significant phase separations and dislocations during the formation of InGaN alloys [60,61]. Obviously, the well-ordered $\mathrm{SiH}_{x}$ layered structures we discovered here could overcome the large 

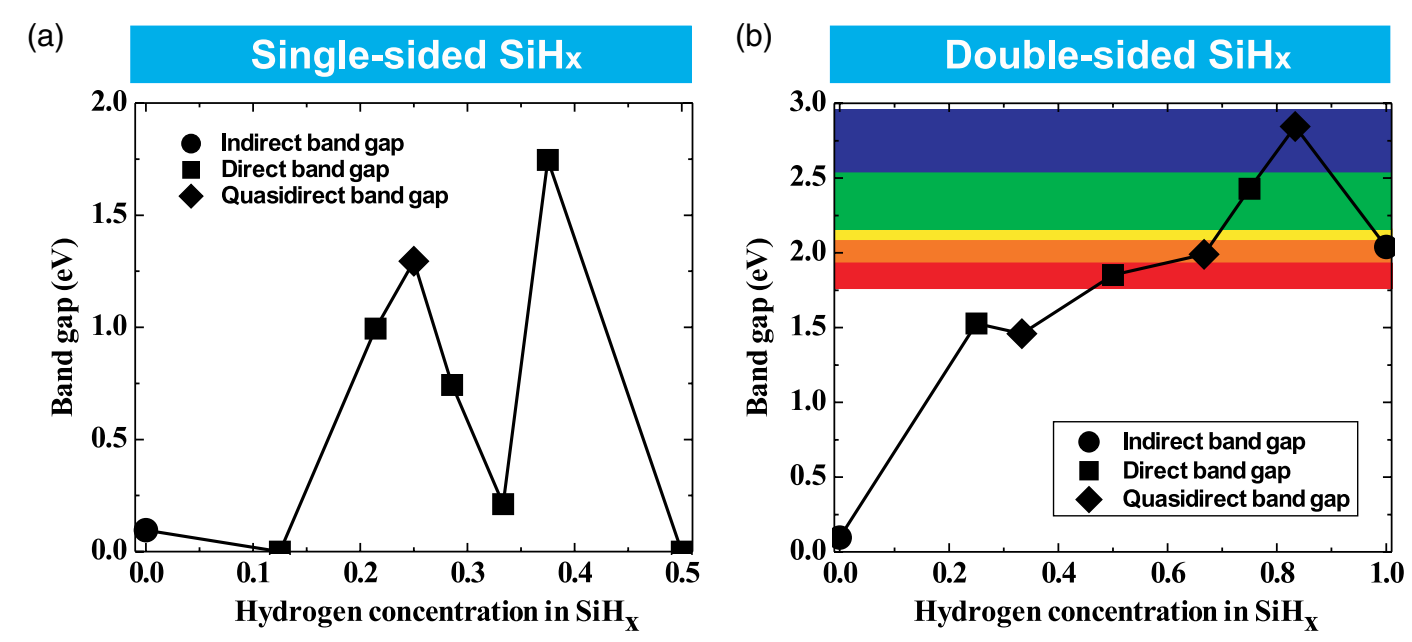

FIG. 5. The calculated band gaps of (a) single-sided $\mathrm{SiH}_{x}$ and (b) double-sided $\mathrm{SiH}_{x}$ ground states as a function of hydrogen concentration. In panel (b), a sketch of light spectrum is overlaid to aid visualization of the size of band gap.

lattice-mismatch issue in InGaN alloys for high-efficiency white lighting. Moreover, it is found that layered materials have unique advantages over the conventional bulk materials for lighting because of the very strong optical transition rates at their band edges $[59,62]$. As far as we know, this is the first report on the discovery of silicon-based materials for white lighting, which can extend our current knowledge of the use of silicon for optoelectronics.

We first attempt to understand the electronic and optical properties of $\mathrm{Si}_{28} \mathrm{H}_{6}, \mathrm{Si}_{24} \mathrm{H}_{6}, \mathrm{Si}_{16} \mathrm{H}_{4}$, and $\mathrm{Si}_{12} \mathrm{H}_{4}$ for solar applications. Figure 6 shows the calculated band structures of single-sided $\mathrm{Si}_{28} \mathrm{H}_{6}$ and $\mathrm{Si}_{24} \mathrm{H}_{6}$. $\mathrm{Si}_{28} \mathrm{H}_{6}$ is a direct-gap semiconductor; i.e., both VBM and $\mathrm{CBM}$ are located at the $\Gamma$ point. The wave function of VBM is contributed mostly by the hybridized $\pi$ and $\sigma$ orbitals of three-folded Si atoms at the bottom layer, while the wave function of CBM is contributed mostly by the $\pi$ orbitals of these special threefolded Si atoms (surrounded by four-folded $\mathrm{Si}$ atoms) at the bottom layer, as shown in Fig. 6(a). The partially localized charge distribution gives rise to a relatively flat top valence band and bottom conduction band of $\mathrm{Si}_{28} \mathrm{H}_{6} . \mathrm{Si}_{24} \mathrm{H}_{6}$ is a quasidirect band-gap material; its global VBM is at the A point, while its global CBM is at the Y point. However, the local VBM at the Y point is only $0.05 \mathrm{eV}$ higher in energy than the global VBM. The plotted wave-function distribution in Fig. 6(b) shows that the VBM at the Y point is contributed mostly by the $\pi$ orbitals of the two neighboring three-folded Si atoms at the bottom layer, and a zigzag wave-function chain is formed; meanwhile, the wave function of $\mathrm{CBM}$ at the $\mathrm{Y}$ point is homogeneously distributed in the bottom Si layer, revealing the hybridized $\pi$ and $3 s$ character, as shown in Fig. 6(b). The partial spatial
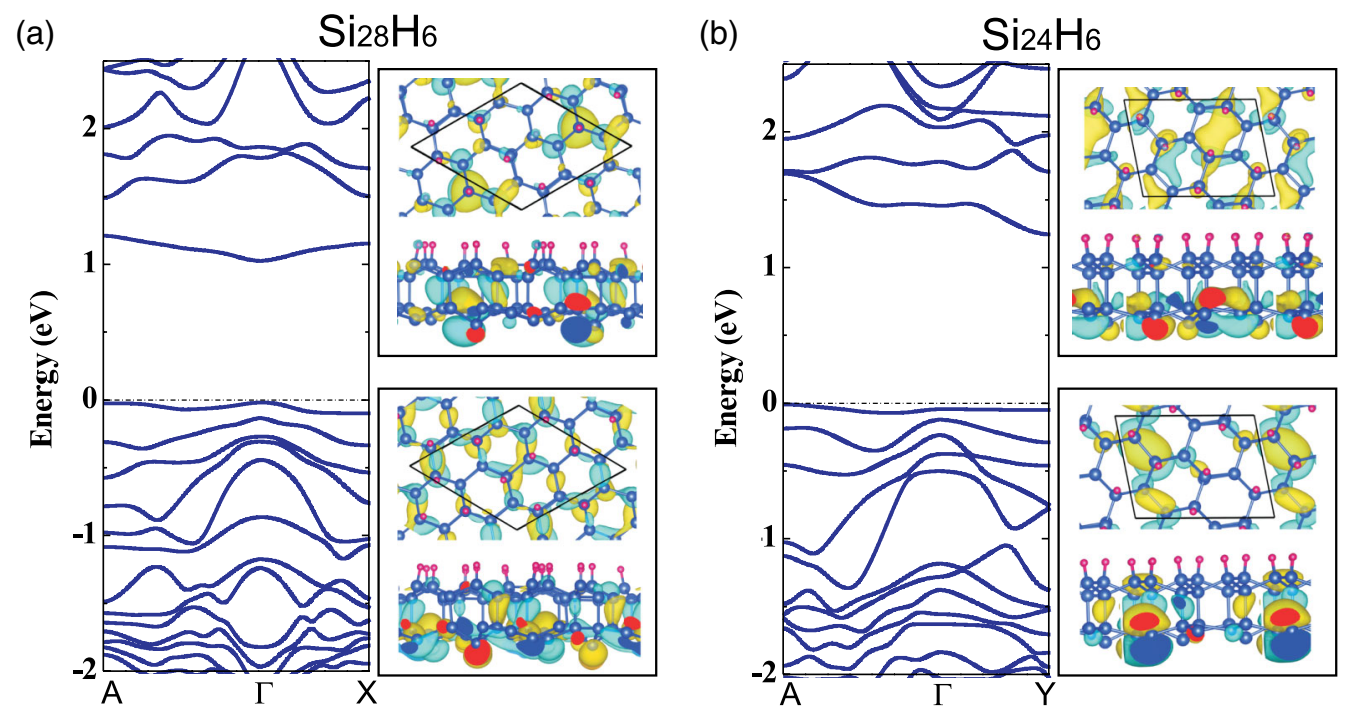

FIG. 6. The calculated band structures of single-sided (a) $\mathrm{Si}_{28} \mathrm{H}_{6}$ and (b) $\mathrm{Si}_{24} \mathrm{H}_{6}$. The wave functions of VBM (bottom figure) and CBM (top figure) at the $\Gamma$ point for $\mathrm{Si}_{28} \mathrm{H}_{6}$ and at the $\mathrm{Y}$ point for $\mathrm{Si}_{24} \mathrm{H}_{6}$ are also plotted here. The Fermi level is set to zero. 

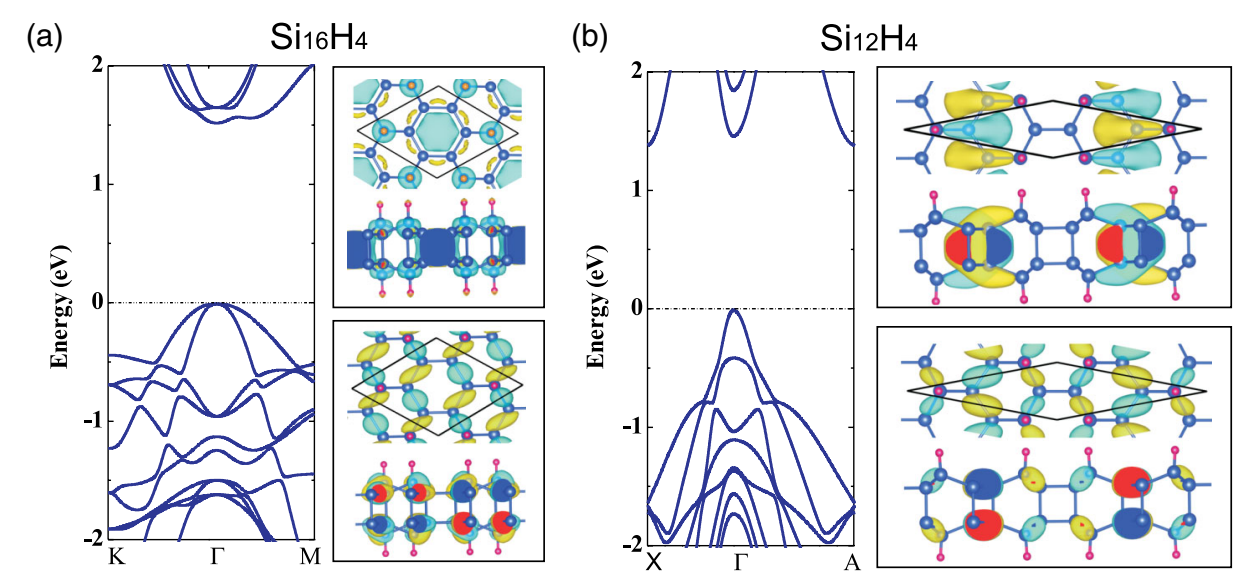

FIG. 7. The calculated band structures of double-sided (a) $\mathrm{Si}_{16} \mathrm{H}_{4}$ and (b) $\mathrm{Si}_{12} \mathrm{H}_{4}$. The wave functions of VBM (bottom figure) and $\mathrm{CBM}$ (top figure) at the $\Gamma$ point for each structure are also plotted here. The Fermi level is set to zero.

localization of the bottom conduction and top valence bands suggests the possibility of electron-hole separation in $\mathrm{Si}_{28} \mathrm{H}_{6}$ and $\mathrm{Si}_{24} \mathrm{H}_{6}$, which might be useful for reducing the carrier recombination during photoexcitation.

Figure 7 shows the calculated band structures of doublesided $\mathrm{Si}_{16} \mathrm{H}_{4}$ and $\mathrm{Si}_{12} \mathrm{H}_{4} . \mathrm{Si}_{16} \mathrm{H}_{4}$ is a direct band-gap semiconductor. Its VBM is contributed by the $\sigma$ orbitals of all the Si atoms. Interestingly, we find that the wave function of CBM is contributed not only by the $3 s$ electrons of these $\mathrm{Si}$ atoms with adsorbed $\mathrm{H}$ atoms, but also by the nearly free-electron (NFE) states, which are localized inside of the benzene-ring-like $\mathrm{Si}$ atoms (without adsorbed $\mathrm{H}$ atoms), as shown in Fig. 7(a). Our further calculations show that the electron effective mass at CBM is around $1.04 \mathrm{~m}_{e}$ (in units of free-electron mass), which is an important feature of NFE states. Because the NFE states are not localized on the lattice site of a material, in principle, it will be less affected by structural defects and, therefore, could realize stable charge transport. It has been reported that NFE states can exist in isolated crystalline carbon sheets and nanotubes [63-66], and this is the first report that NFE states can also exist in silicon materials. The $\mathrm{Si}_{12} \mathrm{H}_{4}$ is a quasidirect semiconductor. The global VBM of this structure is at the $\Gamma$ point, while the global CBM is at the $\mathrm{X}$ point. However, the local CBM at the $\Gamma$ point is only $0.07 \mathrm{eV}$ higher than the global CBM. The wave function of $\mathrm{VBM}$ of $\mathrm{Si}_{12} \mathrm{H}_{4}$ is contributed mostly by the $\sigma$ orbitals of all the $\mathrm{Si}$ atoms, while the wave function of $\mathrm{CBM}$ at the $\Gamma$ point is contributed by the $3 s$ orbitals of these zigzag-chain $\mathrm{Si}$ atoms without $\mathrm{H}$ atoms, as shown in Fig. 7(b). One unique advantage of $\mathrm{Si}_{12} \mathrm{H}_{4}$ for solar applications is that the carrier effective masses are quite small; e.g., the electron and hole effective mass at the $\Gamma$ point are about 0.16 and $0.09 \mathrm{~m}_{e}$, respectively. A small effective mass usually means high carrier mobility, which combines with partial electron-hole separation [Fig. 7(b)], suggesting that $\mathrm{Si}_{12} \mathrm{H}_{4}$ could be a good candidate for highefficiency solar cells.
The above results show that $\mathrm{Si}_{28} \mathrm{H}_{6}, \mathrm{Si}_{24} \mathrm{H}_{6}, \mathrm{Si}_{16} \mathrm{H}_{4}$, and $\mathrm{Si}_{12} \mathrm{H}_{4}$ have suitable band gaps in the range of $1.0-1.5 \mathrm{eV}$, which are good for solar-cell applications. To further investigate their suitability, we have calculated the absorption spectra for these four structures, compared to the reference air-mass 1.5-solar-spectral irradiance [67], as shown in Fig. 8. The absorbance $\mathrm{A}(\omega)$ of monolayer HBS is defined as the fraction of photons with energy $\mathrm{E}=\hbar \omega$ absorbed by the HBS $[68,69]$. It can be calculated as

$$
A(\omega)=\frac{\omega}{c} \varepsilon_{2} \Delta z
$$

where $\varepsilon_{2}$ is the calculated imaginary part of the dielectric function [62,70], $c$ is the speed of light, and $\Delta z$ is the size of the supercell in the layer-normal direction. Equation (5) is the approximation of the absorbance $A(\omega)=1-e^{-\alpha \cdot \Delta z}$

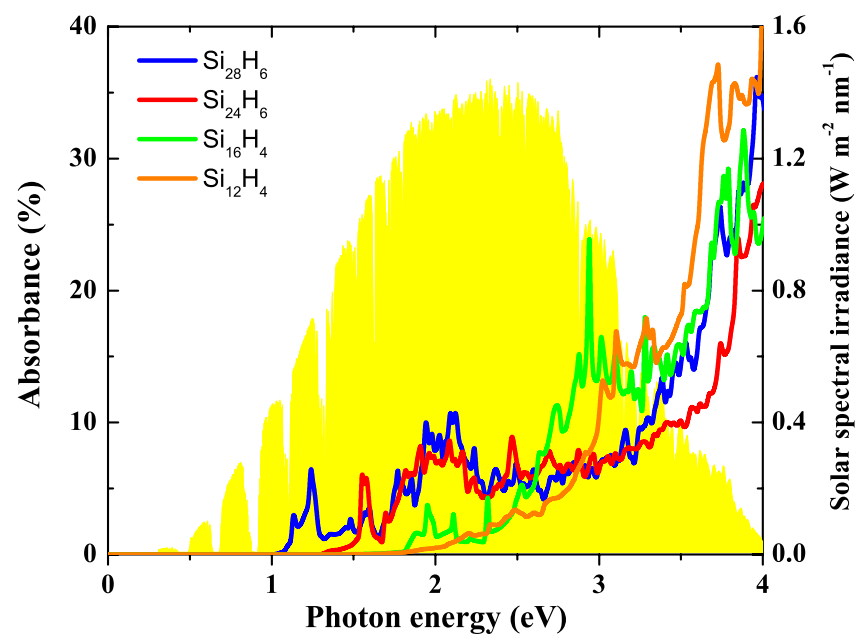

FIG. 8. The calculated optical absorbance spectra of $\mathrm{Si}_{28} \mathrm{H}_{6}$, $\mathrm{Si}_{24} \mathrm{H}_{6}, \mathrm{Si}_{16} \mathrm{H}_{4}$, and $\mathrm{Si}_{12} \mathrm{H}_{4}$. The reference air-mass 1.5-solarspectral irradiance is plotted in yellow. 
(a)

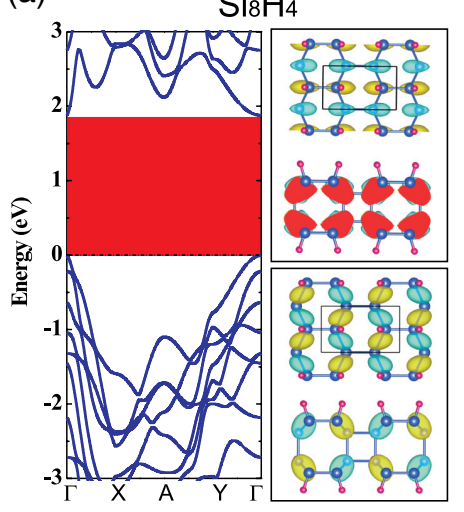

(b)

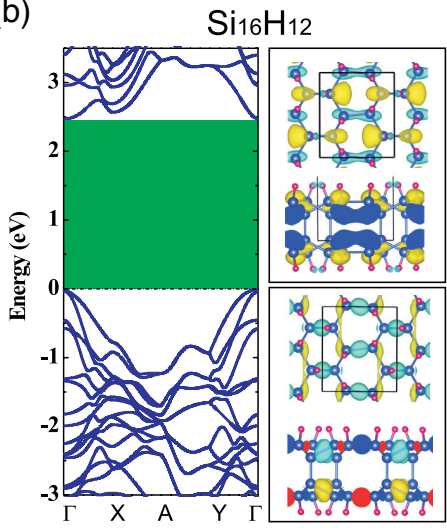

(c)

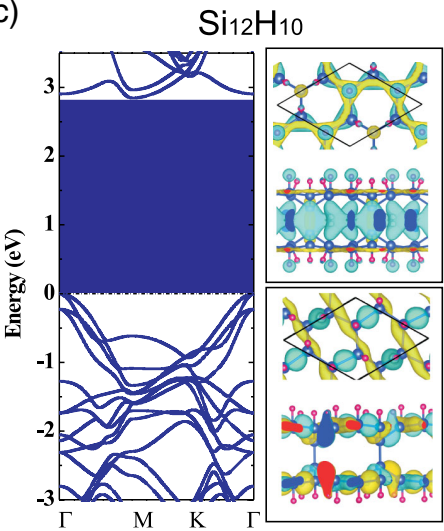

FIG. 9. The calculated band structures of double-sided (a) $\mathrm{Si}_{8} \mathrm{H}_{4}$, (b) $\mathrm{Si}_{16} \mathrm{H}_{12}$, and (c) $\mathrm{Si}_{12} \mathrm{H}_{10}$. The wave functions of VBM (bottom figure) and CBM (top figure) at the $\Gamma$ point for each structure are also plotted here. The color for each band gap is also shown. The Fermi level is set to zero.

$\left(\alpha=\varepsilon_{2} \omega / c n\right)$ for a monolayer material with small thickness $\Delta z$. The monolayer absorbance $\mathrm{A}(\omega)$ is independent of the size of the vacuum layer because $\varepsilon_{2} \propto 1 / \Delta z$ when a vacuum is included to simulate HBS. Then, we can define the flux of absorbed photons $J_{a b s}[69]$ as

$$
J_{a b s}=e \int_{E_{g}}^{\infty} A(E) J_{p h}(E) d E,
$$

where $E_{g}$ is the optical gap of the absorber and $J_{p h}(\mathrm{E})$ is the incident photon flux $[67,69] . J_{a b s}$ can be expressed as the equivalent short-circuit electrical current density in the ideal case when all the photons are converted to carriers and extracted during the $\mathrm{PV}$ process. Our calculations show that the $J_{a b s}$ for $\mathrm{Si}_{28} \mathrm{H}_{6}, \mathrm{Si}_{24} \mathrm{H}_{6}, \mathrm{Si}_{16} \mathrm{H}_{4}$, and $\mathrm{Si}_{12} \mathrm{H}_{4}$ structures are $8.6,7.4,6.9$, and $6.5 \mathrm{~mA} / \mathrm{cm}^{2}$, respectively. Considering that the $J_{a b s}$ of 1-nm-diamond $\mathrm{Si}$ is only $0.1 \mathrm{~mA} / \mathrm{cm}^{2}$ [69], this means that $60-90-\mathrm{nm}$-thick $\mathrm{Si}$ is necessary to absorb the same fraction of sunlight as these subnanometer-thick HBS. Clearly, the use of HBS-based thin-film solar cells can largely reduce the cost of silicon PV modules, which may have the potential to overcome the big drawback of diamond-silicon-based solar cells. The significantly high optical absorbance of HBS structures compared to silicon is mainly due to the narrowed band gaps and dipole-allowed transitions at the band edges. To maximize the solar-cell efficiency, thicker multilayer stacking of these HBS structures in a bulk heterojunction manner may be needed in practice.

Finally, we study the band structures of double-sided $\mathrm{Si}_{8} \mathrm{H}_{4}, \mathrm{Si}_{16} \mathrm{H}_{12}$, and $\mathrm{Si}_{12} \mathrm{H}_{10}$, which are proposed to be candidates for realizing RGB colors for white lighting. As shown in Fig. 9(a), $\mathrm{Si}_{8} \mathrm{H}_{4}$ is a direct band-gap material and its CBM and VBM are both located at the $\Gamma$ point. The VBM wave function of this structure is contributed by the Si $\sigma$ orbitals, while the wave function of CBM [Fig. 9(b)] is contributed by the $3 s$ orbitals of these $\mathrm{Si}$ atoms underneath the surface. Similar to $\mathrm{Si}_{8} \mathrm{H}_{4}$, the wave function of VBM for the structure of $\mathrm{Si}_{16} \mathrm{H}_{12}$ is contributed by $\mathrm{Si} \sigma$ orbitals, and $\mathrm{CBM}$ is contributed by $\mathrm{Si} 3 \mathrm{~s}$ orbitals. $\mathrm{Si}_{12} \mathrm{H}_{10}$ is a quasidirect band-gap semiconductor, as shown in Fig. 9(c); its VBM is located at the $\Gamma$ point, while the global CBM is located at the M point. However, the bottom conduction band at the $\Gamma$ point is only $0.08 \mathrm{eV}$ higher in energy than that of global CBM. As shown in Fig. 9(c), the wave-function characters at CBM and VBM are similar to those of $\mathrm{Si}_{8} \mathrm{H}_{4}$ and $\mathrm{Si}_{16} \mathrm{H}_{12}$. Clearly, these three $\mathrm{SiH}_{x}$ ground states are suitable for lighting emitters to achieve RGB colors. In practice, multilayer stacking of HBS structures may be used to obtain bright emission.

In conclusion, we have found that hydrogenation of bilayer silicene can induce some well-ordered intermediate ground states with excellent optoelectronic properties, as summarized in Figure 10. At low hydrogen concentrations, four $\mathrm{SiH}_{x}$ ground states, i.e., $\mathrm{Si}_{28} \mathrm{H}_{6}, \mathrm{Si}_{24} \mathrm{H}_{6}, \mathrm{Si}_{16} \mathrm{H}_{4}$, and

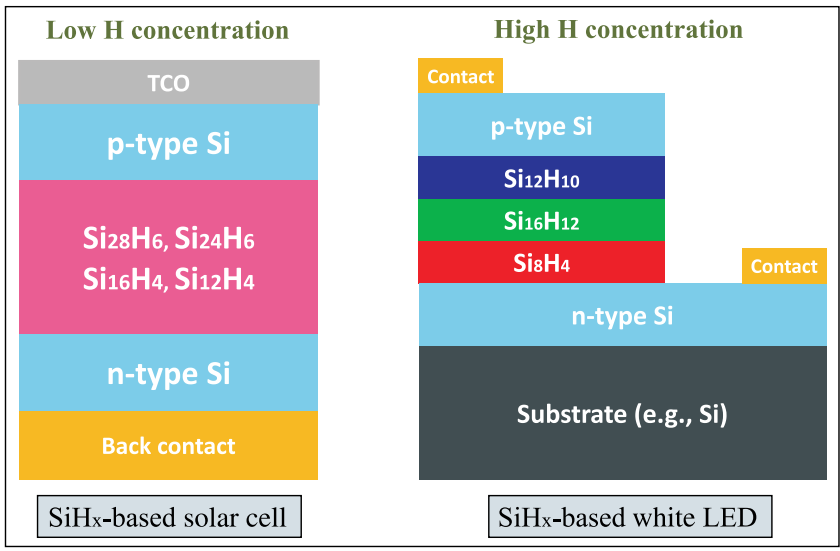

FIG. 10. Summary figure. Unusual optoelectronic properties of hydrogenated bilayer silicon: From solar absorber (low $\mathrm{H}$ concentration) to light-emitting diode (high $\mathrm{H}$ concentration) applications. 
$\mathrm{Si}_{12} \mathrm{H}_{4}$, behave as dipole-allowed direct (or quasidirect) band-gap semiconductors with promising gaps in the range of 1 to $1.5 \mathrm{eV}$, which are suitable for solar PV applications and superior to the current diamond silicon. At high hydrogen concentrations, three well-ordered double-sided $\mathrm{SiH}_{x}$ structures, i.e., $\mathrm{Si}_{8} \mathrm{H}_{4}, \mathrm{Si}_{16} \mathrm{H}_{12}$, and $\mathrm{Si}_{12} \mathrm{H}_{10}$, can have direct (or quasidirect) band gaps within the range of RGB colors, which can be used as light emitters for white LEDs. Therefore, by simply controlling the hydrogen concentration, HBS can potentially be used as new silicon-based solar absorbers, as well as white LEDs.

The research at NREL is sponsored by the U.S. Department of Energy under Contract No. DE-AC3608GO28308. The research at ORNL is sponsored by the the U.S. Department of Energy, Office of Basic Energy Sciences, Materials Sciences and Engineering Division. H. L. is supported by the Basic Science Research Program (Grant No. KRF-2012R1A1A1013124) through the National Research Foundation of Korea, funded by the Ministry of Education, Science and Technology. All the calculations were carried out at the DOE REDMESA and NERSC facilities.

[1] B. P. Jelle, C. Breivik, and H. D. Rokenes, Building Integrated Photovoltaic Products: A State-of-the-Art Review and Future Research Opportunities, Solar Energy Mater. Sol. Cells 100, 69 (2012).

[2] W. Bludau, A. Onton, and W. Heinke, Temperature Dependence of the Band Gap of Silicon, J. Appl. Phys. 45, 1846 (1974).

[3] M. S. Hybertsen and S. G. Louie, First-Principles Theory of Quasiparticles: Calculation of Band Gaps in Semiconductors and Insulators, Phys. Rev. Lett. 55, 1418 (1985).

[4] P. Lautenschlager, M. Garriga, L. Vina, and M. Cardona, Temperature Dependence of the Dielectric Function and Interband Critical Points in Silicon, Phys. Rev. B 36, 4821 (1987).

[5] R. G. Little and M. J. Nowlan, Crystalline Silicon Photovoltaics: The Hurdle for Thin Films, Prog. Photovoltaics 5, 309 (1997).

[6] C. H. Henry, Limiting Efficiencies of Ideal Single and Multiple Energy Gap Terrestrial Solar Cells, J. Appl. Phys. 51, 4494 (1980).

[7] M. A. Green, K. Emery, Y. Hishikawa, W. Warta, and E. D. Dunlop, Solar Cell Efficiency Tables, Prog. Photovoltaics 21, 1 (2013).

[8] J. L. Shay and J. H. Wernick, Ternary Chalcopyrite Semiconductors: Growth, Electronic Properties, and Applications (Pergamon Press, Oxford, 1975).

[9] P. Jackson, D. Hariskos, E. Lotter, S. Paetel, R. Wuerz, R. Menner, W. Wischmann, and M. Powalla, New World Record Efficiency for Cu(In,Ga)Se2 Thin-Film Solar Cells Beyond 20\%, Prog. Photovoltaics 19, 894 (2011).

[10] T. K. Todorov, K. B. Reuter, and D. B. Mitzi, HighEfficiency Solar Cell with Earth-Abundant LiquidProcessed Absorber, Adv. Mater. 22, E156 (2010).
[11] S. Chen, A. Walsh, X.-G. Gong, and S.-H. Wei, Classification of Lattice Defects in the Kesterite Cu2ZnSnS4 and Cu2ZnSnSe4 Earth-Abundant Solar Cell Absorbers, Adv. Mater. 25, 1522 (2013).

[12] W. Shockley and H. J. Queisser, Detailed Balance Limit of Efficiency of $p$ - $n$ Junction Solar Cells, J. Appl. Phys. 32, 510 (1961).

[13] H. J. Xiang, B. Huang, E. Kan, S.-H. Wei, and X.-G. Gong, Towards Direct-Gap Silicon Phases by the Inverse Band Structure Design Approach, Phys. Rev. Lett. 110, 118702 (2013).

[14] S. Botti, J. A. Flores-Livas, M. Amsler, S. Goedecker, and M. A. L. Marques, Low-Energy Silicon Allotropes with Strong Absorption in the Visible for Photovoltaic Applications, Phys. Rev. B 86, 121204 (2012).

[15] T. Watkins, A. V. G. Chizmeshya, L. Jiang, D. J. Smith, R. T. Beeler, G. Grzybowski, C. D. Poweleit, J. Menendez, and J. Kouvetakis, Nanosynthesis Routes to New Tetrahedral Crystalline Solids: Silicon-like Si3AlP, J. Am. Chem. Soc. 133, 16212 (2011).

[16] J.-H. Yang, Y. Zhai, H. Liu, H. J. Xiang, X. G. Gong, and S.-H. Wei, Si3AlP: A New Promising Material for Solar Cell Absorber, J. Am. Chem. Soc. 134, 12653 (2012).

[17] M. Avezac, J.-W. Luo, T. Chanier, and A. Zunger, GeneticAlgorithm Discovery of a Direct-Gap and Optically Allowed Superstructure from Indirect-Gap Si and Ge Semiconductors, Phys. Rev. Lett. 108, 027401 (2012).

[18] M. R. A. Monazam, K. Hingerl, and P. Puschnig, Quasiparticle Band Structure and Optical Properties of the 12 Si-Ge Superstructure from First Principles, Phys. Rev. B 88, 075314 (2013).

[19] D. Li, L. Lin, and J. Feng, Electronic State and Momentum Matrix of H-Passivated Silicon Nanonets: A FirstPrinciples Calculation, Physica (Amsterdam) 42E, 1583 (2010).

[20] S. B. Fagan, R. J. Baierle, R. Mota, Z. J. R. da Silva, and A. Fazzio, Ab Initio Calculations for a Hypothetical Material: Silicon Nanotubes, Phys. Rev. B 61, 9994 (2000).

[21] G. G. Guzman-Verri and L. C. L. Y. Voon, Electronic Structure of Silicon-Based Nanostructures, Phys. Rev. B 76, 075131 (2007).

[22] S. Cahangirov, M. Topsakal, E. Akturk, H. Sahin, and S. Ciraci, Two-and One-Dimensional Honeycomb Structures of Silicon and Germanium, Phys. Rev. Lett. 102, 236804 (2009).

[23] P. Vogt, P. De Padova, C. Quaresima, J. Avila, E. Frantzeskakis, M. C. Asensio, A. Resta, B. Ealet, and G. Le Lay, Silicene: Compelling Experimental Evidence for Graphenelike Two-Dimensional Silicon, Phys. Rev. Lett. 108, 155501 (2012).

[24] B. Feng, Z. Ding, S. Meng, Y. Yao, X. He, P. Cheng, L. Chen, and $\mathrm{K}$. Wu, Evidence of Silicene in Honeycomb Structures of Silicon on Ag(111), Nano Lett. 12, 3507 (2012).

[25] L. Chen, C. Liu, B. Feng, X. He, P. Cheng, Z. Ding, S. Meng, Y. Yao, and K. Wu, Evidence for Dirac Fermions in a Honeycomb Lattice Based on Silicon, Phys. Rev. Lett. 109, 056804 (2012).

[26] P. De Padova, O. Kubo, B. Olivieri, C. Quaresima, T. Nakayama, M. Aono, and G. Le Lay, Multilayer Silicene Nanoribbons, Nano Lett. 12, 5500 (2012). 
[27] C.-L. Lin, R. Arafune, K. Kawahara, M. Kanno, N. Tsukahara, E. Minamitani, Y. Kim, M. Kawai, and N. Takagi, Substrate-Induced Symmetry Breaking in Silicene, Phys. Rev. Lett. 110, 076801 (2013).

[28] A. Resta, T. Leoni, C. Barth, A. Ranguis, C. Becker, T. Bruhn, P. Vogt, and G. Le Lay, Atomic Structures of Silicene Layers Grown on Ag(111): Scanning Tunneling Microscopy and Noncontact Atomic Force Microscopy Observations, Sci. Rep. 3, 2399 (2013).

[29] R. Arafune, C.-L. Lin, K. Kawahara, N. Tsukahara, E. Minamitani, Y. Kim, N. Takagi, and M. Kawai, Structural Transition of Silicene on Ag(111), Surf. Sci. 608, 297 (2013).

[30] P. De Padova, J. Avila, A. Resta, I. Razado-Colambo, C. Quaresima, C. Ottaviani, B. Olivieri, T. Bruhn, P. Vogt, M. C. Asensio, and G. Le Lay, The Quasiparticle Band Dispersion in Epitaxial Multilayer Silicene, J. Phys. Condens. Matter 25, 382202 (2013).

[31] P. De Padova, P. Vogt, A. Resta, J. Avila, I. RazadoColambo, C. Quaresima, C. Ottaviani, B. Olivieri, T. Bruhn, T. Hirahara, T. Shirai, S. Hasegawa, M. C. Asensio, and G. Le Lay, Evidence of Dirac Fermions in Multilayer Silicene, Appl. Phys. Lett. 102, 163106 (2013).

[32] P. Vogt, P. Capiod, M. Berthe, A. Resta, P. De Padova, T. Bruhn, G. Le Lay, and B. Grandidier, Synthesis and Electrical Conductivity of Multilayer Silicene, Appl. Phys. Lett. 104, 021602 (2014).

[33] L. Meng, Y. Wang, L. Zhang, S. Du, R. Wu, L. Li, Y. Zhang, G. Li, H. Zhou, W. A. Hofer, and H.-J. Gao, Buckled Silicene Formation on Ir(111), Nano Lett. 13, 685 (2013).

[34] A. Fleurence, R. Friedlein, T. Ozaki, H. Kawai, Y. Wang, and Y. Yamada-Takamura, Experimental Evidence for Epitaxial Silicene on Diboride Thin Films, Phys. Rev. Lett. 108, 245501 (2012).

[35] F. Liu, C. Liu, K. Wu, F. Yang, and Y. Yao, $d+$ id Chiral Superconductivity in Bilayer Silicene, Phys. Rev. Lett. 111, 066804 (2013).

[36] M. Ezawa, Quasi-Topological Insulator and Trigonal Warping in Gated Bilayer Silicene, J. Phys. Soc. Jpn. 81, 104713 (2012).

[37] G. A. Tritsaris, E. Kaxiras, S. Meng, and E. Wang, Adsorption and Diffusion of Lithium on Layered Silicon for Li-Ion Storage, Nano Lett. 13, 2258 (2013).

[38] C. Lian and J. Ni, Strain Induced Phase Transitions in Silicene Bilayers: A First Principles and Tight-Binding Study, AIP Adv. 3, 052102 (2013).

[39] C. Kamal, A. Chakrabarti, A. Banerjee, and S. K. Deb, Silicene Beyond Mono-layers Different Stacking Configurations and Their Properties, J. Phys. Condens. Matter 25, 085508 (2013).

[40] S. Ryu, M. Y. Han, J. Maultzsch, T. F. Heinz, P. Kim, M. L. Steigerwald, and L.E. Brus, Reversible Basal Plane Hydrogenation of Graphene, Nano Lett. 8, 4597 (2008).

[41] D. C. Elias, R. R. Nair, T. M. G. Mohiuddin, S. V. Morozov, P. Blake, M. P. Halsall, A. C. Ferrari, D. W. Boukhvalov, M. I. Katsnelson, A. K. Geim, and K. S. Novoselov, Control of Graphene's Properties by Reversible Hydrogenation: Evidence for Graphane, Science 323, 610 (2009).

[42] R. Balog, B. Jogensen, L. Nilsson, M. Andersen, E. Rienks, M. Bianchi, M. Fanetti, E. Lagsgaard, A. Baraldi, S. Lizzit,
Z. Sljivancanin, F. Besenbacher, B. Hammer, T. G. Pedersen, P. Hofmann, and L. Hornekar, Bandgap Opening in Graphene Induced by Patterned Hydrogen Adsorption, Nat. Mater. 9, 315 (2010).

[43] J. Koo, H. J. Hwang, B. Huang, H. Lee, H. Lee, M. Park, Y. Kwon, S.-H. Wei, and H. Lee, Exotic Geometrical and Electronic Properties in Hydrogenated Graphyne, J. Phys. Chem. C 117, 11960 (2013).

[44] J. C. Garcia, D. B. Lima, L. V. C. Assali, and J. F. Justo, Group IV Graphene-and Graphane-Like Nanosheets, J Phys. Chem. C 115, 13242 (2011).

[45] M. Houssa, E. Scalise, K. Sankaran, G. Pourtois, V. V. Afanasev, and A. Stesmans, Electronic Properties of Hydrogenated Silicene and Germanene, Appl. Phys. Lett. 98, 223107 (2011).

[46] B. Huang, H. J. Xiang, and S. -H. Wei, Chemical Functionalization of Silicene: Spontaneous Structural Transition and Exotic Electronic Properties, Phys. Rev. Lett. 111, 145502 (2013).

[47] G. Kresse and J. Furthmüller, Efficiency of Ab-Initio Total Energy Calculations for Metals and Semiconductors Using a Plane-Wave Basis Set, Comput. Mater. Sci. 6, 15 (1996).

[48] S. Grimme, Semiempirical GGA-Type Density Functional Constructed with a Long-Range Dispersion Correction, J. Comput. Chem. 27, 1787 (2006).

[49] G. Mercurio, E. R. McNellis, I. Martin, S. Hagen, F. Leyssner, S. Soubatch, J. Meyer, M. Wolf, P. Tegeder, F. S. Tautz, and K. Reuter, Structure and Energetics of Azobenzene on Ag(111): Benchmarking Semiempirical Dispersion Correction Approaches, Phys. Rev. Lett. 104, 036102 (2010).

[50] B. Huang, Q. Xu, and S.-H. Wei, Theoretical Study of Corundum as an Ideal Gate Dielectric Material for Graphene Transistors, Phys. Rev. B 84, 155406 (2011).

[51] J. Heyd, G. E. Scuseria, and M. Ernzerhof, Hybrid Functionals Based on a Screened Coulomb Potential, J. Chem. Phys. 118, 8207 (2003).

[52] L. G. Ferreira, S. H. Wei, and A. Zunger, First-Principles Calculation of Alloy Phase Diagrams: The RenormalizedInteraction Approach, Phys. Rev. B 40, 3197 (1989).

[53] B. Huang, H. J. Xiang, Q. Xu, and S.-H. Wei, Overcoming the Phase Inhomogeneity in Chemically Functionalized Graphene: The Case of Graphene Oxides, Phys. Rev. Lett. 110, 085501 (2013).

[54] A. van de Walle, M. Asta, and G. Ceder, The Alloy Theoretic Automated Toolkit: A User Guide, CALPHAD: Comput. Coupling Phase Diagrams Thermochem. 26, 539 (2002).

[55] Y. Wang, J. Lv, L. Zhu, and Y. Ma, Crystal Structure Prediction via Particle-Swarm Optimization, Phys. Rev. B 82, 094116 (2010).

[56] See Supplemental Material at http://link.aps.org/ supplemental/10.1103/PhysRevX.4.021029 for the calculations of bilayer silicon on $\operatorname{Ag}(111)$ substrate.

[57] H. Xiang, E. Kan, S.-H. Wei, M.-H. Whangbo, J. Yang, Narrow Graphene Nanoribbons Made Easier by Partial Hydrogenation, Nano Lett. 9, 4025 (2009).

[58] K. Erickson, R. Erni, Z. Lee, N. Alem, W. Gannett, and A. Zettl, Determination of the Local Chemical Structure of Graphene Oxide and Reduced Graphene Oxide, Adv. Mater. 22, 4467 (2010). 
[59] K. Watanabe, T. Taniguchi, and H. Kanda, Direct-Bandgap Properties and Evidence for Ultraviolet Lasing of Hexagonal Boron Nitride Single Crystal, Nat. Mater. 3, 404 (2004).

[60] A. Bergh, G. Craford, A. Duggal, and R. Haitz, The Promise and Challenge of Solid-State Lighting, Phys. Today 54, No. 1242 (2001).

[61] L. Bergman and J. L. McHale, Handbook of Luminescent Semiconductor Materials (Taylor Francis Group, Boca Raton, 2011).

[62] B. Huang, X. K. Cao, H. X. Jiang, J. Y. Lin, and S.-H. Wei, Origin of the Significantly Enhanced Optical Transitions in Layered Boron Nitride, Phys. Rev. B 86, 155202 (2012).

[63] X. Blase, A. Rubio, S. G. Louie, and M. L. Cohen, Quasiparticle Band Structure of Bulk Hexagonal Boron Nitride and Related Systems, Phys. Rev. B 51, 6868 (1995).

[64] M. Posternak, A. Baldereschi, A. J. Freeman, E. Wimmer, and M. Weinert, Prediction of Electronic Interlayer States in Graphite and Reinterpretation of Alkali Bands in Graphite Intercalation Compounds, Phys. Rev. Lett. 50, 761 (1983).
[65] E. R. Margine and V.H. Crespi, Universal Behavior of Nearly Free Electron States in Carbon Nanotubes, Phys. Rev. Lett. 96, 196803 (2006).

[66] S. Hu, J. Zhao, Y. Jin, J. Yang, H. Petek, and J. G. Hou, Nearly Free Electron Superatom States of Carbon and Boron Nitride Nanotubes, Nano Lett. 10, 4830 (2010).

[67] See NREL website: http://rredc.nrel.gov/solar/spectra/ am1.5.

[68] L. Yang, J. Deslippe, C. -H. Park, M. L. Cohen, and S. G. Louie, Excitonic Effects on the Optical Response of Graphene and Bilayer Graphene, Phys. Rev. Lett. 103, 186802 (2009).

[69] M. Bernardi, M. Palummo, and J. C. Grossman, Extraordinary Sunlight Absorption and One Nanometer Thick Photovoltaics Using Two-Dimensional Monolayer Materials, Nano Lett. 13, 3664 (2013).

[70] M. Gajdos, K. Hummer, G. Kresse, J. Furthmuller, and F. Bechstedt, Linear Optical Properties in the ProjectorAugmented Wave Methodology, Phys. Rev. B 73, 045112 (2006). 